Renata S. S. Guizzardi, Virginia Dignum, Anna Perini, Gerd Wagner: Towards an Integrated Methodology to Develop KM Solutions with the Support of Agents, Proceedings of KIMAS'05, IEEE, Waltham, Massachusetts, 8 - 21 April 2005. 


\title{
Towards an Integrated Methodology to Develop KM Solutions with the Support of Agents
}

\author{
Renata S. S. Guizzardi ${ }^{1}$, Virginia Dignum², \\ Gerd Wagner $^{3}$, Anna Perini ${ }^{4}$, Pablo G. Ludermir ${ }^{1}$ \\ ${ }^{1}$ Computer Science Department, University of Twente \\ P.O. Box 217 - 7500 AE Enschede - The Netherlands \\ \{souza,pablo.gomes\}@cs.utwente.nl \\ ${ }^{2}$ Institute of Information and Computing Sciences, Utrecht University \\ P.O. Box 80089 - 3508 TB Utrecht - The Netherlands \\ virginia@cs.uu.nl \\ ${ }^{3}$ Department of Information \& Technology, Eindhoven University of Technology (TU/e) \\ P.O. Box 513 - 5600 MB Eindhoven - The Netherlands \\ G.Wagner@tm.tue.nl \\ ${ }^{4}$ ITC-irst \\ via Sommarive 18, I-38050, Trento-Povo, Italy \\ perini@itc.it
}

Organizational processes have gone through profound changes in the past years, becoming more dynamic and knowledge intensive. These changes have been necessary for businesses to maintain sustainable advantage on the market. On the other hand, the new business models brought about a wide variety of problems to be solved, and a solution is many times based on the development of new network based systems. In parallel, the software engineering field faces a big challenge in providing the right kinds of abstractions and methods to model such systems.

In this work, we argue that agents are suitable for modeling human and artificial organizations due to their autonomous, reactive and proactive nature. Moreover, agent's cognitive characteristics such as goals, plans, beliefs and commitments are adequate constructs to capture and describe human behavior and organizational norms and processes. However, having the right abstraction is not enough for guaranteeing the development of adequate solutions for the organization. For that, a consistent software engineering methodology is needed.

Here, we present preliminary results of our work on an integrated agent-oriented methodology to develop Knowledge Management solutions. This methodology comprehends the software engineering phases of analysis and design, proposing a cyclic development process that iterates through these phases.

Innovation in our work derives from the fact that we consider all stakeholders (organizations and humans) as agents in our analysis model, and start by understanding their relations before actually thinking of developing a system. Perhaps the problem may be more effectively solved by proposing changes in the business processes, rather than by making use of technology. Or perhaps the system is needed but requires process changes as well. Both conclusions are possible outcomes of our analysis. Besides, in addition to humans and organizations, existing systems are also included in the model from start, 
helping us understand which functionalities are delegated to these so called artificial agents. Hence, the new system may be developed on top of legacy solutions, or in other words, the new agent(s) may interact with these systems as external agents that will meet some goals on its (their) behalf.

Benefits as a result of the application of this methodology may be also attributed to our choice of using the proper agent cognitive characteristics in the different phases of the development cycle. Concepts such as agent's beliefs, goals, and plans are vastly discussed in literature and different models have been proposed. However, it is hard to know how to go from theory to practice. In this respect, our work attempts to provide an answer to the following questions: Should these concepts be considered all at once in system development? If not, when are goals suitable, and when should the developer start considering agent's beliefs? And, perhaps, the most frequent question of all: How can these concepts be materialized in practical elements of a program? Such questions have crossed the minds of many software developers when using agents on system design. Although there is no final answer for them, this work aims at contributing to clarify these important issues, by presenting a consistent process on how to go from requirements analysis until detailed design, which will finally lead the system to be developed and deployed.

This paper shows how the methodology may be used in the development of a KM system. We build on top of previous publications that target the initial phases of system development, focusing here on the system design phases. Although the KM domain is here used to exemplify our approach, it is our belief that the proposed methodology may be generalized to solve other kinds of problems within organizational settings. 